Method. The study is a descriptive cross-sectional study conducted among students of randomly selected tertiary institutions in south western Nigeria. Ethical approval was obtained from the Research and Ethics Commitee of the Federal Neuropsychiatric Hospital Abeokuta Ogun State Nigeria. Permission to carry out the study was sought from the University authorities. A multistage cluster sampling selection of 850 respondents was done. Consenting students were administered socio-demographic questionnaire, WHO student's drug use questionnaire, the Big Five Personality Inventory (BFI-44), perceived stress scale-10 and academic motivation inventory.

Result. Seven hundred and eighty one completed questionnaires were analysed yielding a response rate of $92 \%$. There were $51 \%$ males and $49 \%$ females with a mean age of 23.3 years $(\mathrm{SD}=$ \pm 2.29 ), from monogamous family setting 591(75\%) and high socio-economic class $(65.8 \%)$. Of the respondents, $24.8 \%$ reported experience of use related harmful consequences such as engaging in quarrel or argument, unprotected sex and sex regretted the next day. There were significant associations between male gender $(\mathrm{p}=<0.001)$, urban residence $(\mathrm{p}=0.028)$, polygamous family setting $(p=0.002)$, high socioeconomic status $(p=0.026)$ and use related harmful consequences.

Multiple logistic regression showed that the odds of experiencing harmful consequences was less than 1 for agreeableness $(\mathrm{OR}=0.515, \mathrm{df}=1, \mathrm{p}=<0.001)$ and openness $(\mathrm{OR}=0.634, \mathrm{df}=1$, $\mathrm{p}=<0.028)$ but greater than 1 for extraversion $(\mathrm{OR}=1.525$, $\mathrm{df}=1, \mathrm{p}=<0.036$ ) personality dimensions. This implies that for a unit increase in agreeableness and openness scores, there were decreased odds $(8.6 \%$ and $79 \%$ respectively) of experiencing harmful consequences while there was increased odd (86\%) of experiencing harmful consequences from a unit increase in extraversion score.

Both binary and multiple regression analysis revealed that the odds of experiencing harmful consequences is greater than 1 for perceived stress score $(\mathrm{OR}=1.079, \mathrm{p}=<0.001)$ and less than 1 for academic motivation $(\mathrm{OR}=0.975, \mathrm{p}=<0.001)$. This means that perceived stress is positively associated with substance use and experience of harmful consequences while academic motivation is negatively associated with substance use and experience of harmful consequences

Conclusion. There were associations between certain sociodemographic factors, personality dimensions, stress perception and academic motivation with substance use and experience of harmful consequences. Thus, clinicians and researchers should consider these factors when designing preventive and treatment strategies.

\section{A narrative literature review of the typology of psychiatric emergency services in the UK \\ Dhruba Bagchi ${ }^{1 \star}$, George Tadros ${ }^{2}$ and Opeyemi Odejimi ${ }^{1}$ \\ ${ }^{1}$ Birmingham and Solihull Mental Health NHS Foundation Trust and ${ }^{2}$ Birmingham and Solihull Mental Health NHS Foundation Trust, Aston University \\ ${ }^{*}$ Corresponding author.}

doi: 10.1192/bjo.2021.629

Aims. This study aims to provide a detailed literature review of the different forms of Psychiatric Emergency Services currently available within the UK.

Background. 1 in 6 individuals have one form of mental health disorders. Mental health crisis resulting in an individual requiring access to Psychiatric Emergency Service (PES) can occur at any time. Psychiatric Emergency Service (PES) is described as one that provides an immediate response to an individual in crisis within the first 24 hours. Presently, several PESs are available in the UK with the aim of providing prompt and effective assessment, management and in some cases treatment and/or referral. Over the years, economic and political influences have greatly determined the service delivery models of PES. Indeed, these services vary in name, accessibility, structure, professionals involved, outcomes and many more.

Method. Electronic search of five key databases (MEDLINE, PsychINFO, EMBASE, AMED and PUBMED) was carried out to identify various models of PES in the UK. Various combinations of search terms were used and studies which met the inclusion criteria were selected. Studies were included if they were written in English, conducted within the United Kingdom, and described a form of PES. Search was not limited by years and this is to help have a comprehensive overview as well as show changes over time of the various models of psychiatric emergency services. Studies which did not meet any of the criteria detailed above were excluded.

Result. In total, 59 relevant studies were found which identified nine type of PES-Crisis resolution home treatment, police officer intervention, street triage, mental health liaison services in the Emergency Department, psychiatric assessment unit, integrated services, voluntary services and crisis house. There were more papers describing Crisis resolution home treatment services than the others. Furthermore, majority of the papers reported services within England than other countries within the UK.

Conclusion. All forms of PES are beneficial, particularly to mental health service users, but not without some shortcomings. There is a need to continue carrying out methodological research that evaluate impact, cost-effectiveness as well as identify methods of optimising the beneficial outcomes of all models of PES. This will inform researchers, educationist, policy makers and commissioners, service users and carers, service providers and many more on how to ensure current and future PES meet the needs as well as aid recovery of mental health service users.

\section{Mental wellbeing in doctors: the measure matters! development of a core outcome set for measuring wellbeing in doctors}

David Baldwin ${ }^{1 \star}$, Aimee O’Neill ${ }^{2}$, Julia Sinclair ${ }^{1}$ and Gemma Simons ${ }^{2}$

${ }^{1}$ Professor of Psychiatry and Head of Mental Health Group, University of Southampton Faculty of Medicine Honorary Professor of Psychiatry, University of Cape Town, South Africa Visiting Professor, Shandong Mental Health Centre, China and ${ }^{2}$ Centre for Workforce Wellbeing, University of southampton ${ }^{\star}$ Corresponding author.

doi: 10.1192/bjo.2021.630

Aims. To achieve a consensus Core Outcome Set for measuring mental wellbeing in doctors.

Hypothesis: A minimum set of valid, reliable and practical wellbeing measures is needed for doctors.

Background. The importance of doctors' mental wellbeing to everyone using Health Care is highlighted by the levels of burnout reported in doctors around the world. In 2019 a number of UK policy documents made recommendations for the wellbeing of doctors, but how those wellbeing interventions are evaluated needs to be defined. Core Outcome Sets are increasingly being used in medicine to prevent waste in research, by recommending the inclusion of a minimum set of valid, reliable and practical measures. An 